\title{
Le déjà-là et le presque-là
}

Ancrage textuel et procédés d'écriture narrative à l'école élémentaire française

"Before and under the text." Writing narratives at French primary school: The influence of reading expert and peer texts

\section{Brigitte Marin et Jacques Crinon}

\section{(2) OpenEdition}

\section{Journals}

Édition électronique

URL : http://journals.openedition.org/pratiques/3364

DOI : $10.4000 /$ pratiques.3364

ISSN : 2425-2042

Éditeur

Centre de recherche sur les médiations (CREM)

Référence électronique

Brigitte Marin et Jacques Crinon, «Le déjà-là et le presque-là », Pratiques [En ligne], 173-174 | 2017, mis en ligne le 10 mars 2017, consulté le 14 novembre 2019. URL : http://journals.openedition.org/ pratiques/3364; DOI : 10.4000/pratiques.3364

Ce document a été généré automatiquement le 14 novembre 2019.

(C) Tous droits réservés 


\title{
Le déjà-là et le presque-là
}

\author{
Ancrage textuel et procédés d'écriture narrative à l'école élémentaire \\ française \\ "Before and under the text." Writing narratives at French primary school: The \\ influence of reading expert and peer texts
}

Brigitte Marin et Jacques Crinon

1 L'expérience de lecture et d'écriture scolaire et extrascolaire des élèves constitue un socle de connaissances " déjà-là », acquises dans des contextes de socialisation différenciés, qui peuvent leur permettre - à certaines conditions d'accompagnement et d'étayage d'accéder à des registres de connaissances instituées et de pratiques symboliques associées à l'univers langagier de l'école (Penloup, 2007). Les travaux portant sur les pratiques langagières requises dans le champ scolaire ont mis en évidence l'insuffisance d'une approche purement linguistique pour appréhender les productions des élèves et en particulier de ceux dont les usages langagiers diffèrent le plus des constructions litéraciées qu'attend l'école :

Ces constructions, dans leur dimension langagière, relèvent de genres peu familiers pour certains élèves. Mais il s'agit là d'un domaine de recherche encore faiblement exploré, il est peut-être celui qui permettrait aux chercheurs, plus de trente ans après avoir ouvert la question du rôle du langage dans la différenciation sociale et scolaire, de changer la nature de leurs réponses : moins centrées sur les raisons de cette différenciation et davantage sur les moyens d'y remédier. (Bautier, 2001, p. 155)

2 C'est dans une telle perspective que nous analysons des dispositifs élaborés en contexte écologique, pour les besoins de la recherche, afin d'être en prise avec la réalité des classes et de favoriser le transfert de la recherche vers des propositions pour l'enseignement.

3 Des élèves de quatre classes de la fin de l'enseignement primaire ont écrit et réécrit, à quatre reprises au cours d'une même année, des épisodes de romans d'aventures. Ce travail d'écriture allait de pair avec des lectures. Il s'insérait en effet dans des activités de lecture de romans appartenant au même genre, ces romans étant de plus les points de départ des moments d'écriture (l'épisode à écrire s'enchâssait dans le roman en cours de 
lecture). En outre, les élèves conduisaient une correspondance par messagerie électronique avec des partenaires d'une autre classe : les uns lisaient les productions de leurs pairs et leur donnaient des conseils entre la première version et la seconde, les autres recevaient ces conseils.

Cette recherche a fait l'objet de plusieurs publications antérieures, qui ont comparé les performances des élèves qui donnent et de ceux qui reçoivent des conseils, lorsqu'ils écrivent et révisent des récits de fiction, mais aussi des textes explicatifs scientifiques (Crinon, 2012 ; Crinon \& Marin, 2010a et b), qui ont envisagé la place des contraintes liées au genre discursif et à la prise en compte des caractéristiques de celui-ci (Marin \& Crinon, 2014 ; Richard-Principalli, Ferone \& Crinon, 2012), qui ont étudié les corpus du point de vue du caractère implicite ou visible des savoirs textuels de référence (Crinon \& Marin, 2012), ou qui ont analysé le rôle de l'instrumentation technologique dans cette situation de lecture-écriture (Crinon \& Marin, 2010a).

5 La problématique proposée dans ce numéro de Pratiques est l'occasion d'interroger notre corpus sous un angle nouveau. Nous étudions ici la manière dont ces élèves s'appuient sur le « déjà-là » de leurs compétences présentes et s'emparent du « déjà-là » que constituent les mots, les tournures et les connaissances issues des textes qu'ils lisent - textes d'experts et de pairs - pour écrire et récrire leur propre texte, progresser dans leurs compétences narratives, s'approprier les caractéristiques du genre pratiqué.

\section{Déjà-là des ressources textuelles, déjà-là des compétences en construction}

\section{Écriture, lecture, dialogisme}

6 L'idéologie scolaire de la rédaction de textes, ainsi que les conceptions de beaucoup d'élèves, baigne dans la doxa romantique de l'inspiration et de l'expression de soi : avoir des idées (ou ne pas en avoir), exprimer son expérience, ses sentiments, dans leur unicité et leur originalité (ou n'avoir rien à exprimer). Écrire est un don des muses, l'élève (ainsi que son professeur) ne peut que constater qu'il est doué ou qu'il ne l'est pas. À cette croyance s'oppose la conception (dominante chez les écrivains de l'époque classique par exemple) que la création est le fruit d'un travail patient d'imitation, artisanat des mots et reprise des thèmes, des motifs, des procédés, des genres légués par les prédécesseurs.

7 Dans le contexte didactique, cette conception optimiste, qui ne laisse pas le hasard seul maitre des progrès des élèves, joue sur les interactions entre la lecture et l'écriture et peut s'appuyer sur différentes analyses des processus de la production écrite issues de plusieurs approches disciplinaires. Le rôle de la lecture est particulièrement mis en avant dans le modèle cognitif de la production proposé par J. Hayes (1996). Il s'agit d'abord de la lecture ou de la relecture des versions précédentes considérée comme « une composante centrale de la révision» (ibid., p. 82), une lecture-évaluation destinée à détecter des problèmes ou à découvrir des alternatives et des possibilités d'amélioration : lecture de son propre texte ou, dans le cas d'une révision collaborative, lecture du texte d'un partenaire d'écriture. Mais « outre la lecture pour évaluer, deux autres types de lecture jouent des rôles importants dans la rédaction: la lecture de textes sources et la lecture pour définir des tâches " (ibid.). Comprendre et définir la tâche est une condition de l'adéquation du texte produit à ce qui est attendu. Lire est enfin une source de l'écriture, 
lorsqu'on se documente et qu'on emprunte à des textes des connaissances relatives à leur contenu, des idées, mais aussi des mots, des formes linguistiques, rhétoriques et textuelles.

8 Certes les ressources pour écrire, dans le modèle de J.Hayes, sont d'abord les connaissances disponibles en mémoire à long terme (schémas de tâches, connaissance du thème, connaissance du destinataire, connaissance linguistique, connaissance du genre). Ce que, dans d'autres termes, on pourrait en partie assimiler à une culture des textes, des souvenirs organisés issus de lectures. Le rôle de la lecture dans l'acquisition de ces connaissances est d'ailleurs attesté, y compris pour les connaissances linguistiques (sur l'acquisition du vocabulaire, voir Biemiller \& Slonim, 2001; Sénéchal, 2000; sur l'acquisition de certaines compétences en syntaxe, voir Lee, Krashen \& Gribbons, 1996).

9 Souvent, les lectures immédiates influencent aussi l'écriture et font ressource. C'est particulièrement vrai chez les novices ; nous avons montré ailleurs le rôle que peut jouer, chez ceux-ci, l'usage de bases de données textuelles en tant que mémoires externes (Crinon \& Legros, 2002 ; Crinon \& Marin, 2014 ; Legros, Crinon \& Marin, 2006).

10 Insistons également sur l'importance des compétences stratégiques. L'écriture experte ne se contente pas de restituer les connaissances mémorisées, ou empruntées plus immédiatement à des ressources textuelles. Le passage d'une stratégie de "restitution des connaissances » à une stratégie de «transformation des connaissances » est considéré par M. Scardamalia et C. Bereiter (1987) comme une étape que franchit l'apprenti scripteur dans son cheminement vers l'expertise (voir aussi Hayes, 2011). Dans le premier cas, le scripteur « dit » ou « exprime » ses connaissances telles qu'elles se présentent à lui, en particulier en sondant sa mémoire. Dans le second cas, elles sont l'objet d'une réorganisation, d'une mise en ordre, voire d'une réinterprétation au service du projet d'écriture. L'écriture est alors susceptible d'enrichir la connaissance.

11 C'est sans doute alors qu'on peut vraiment parler d'un auteur. Car être auteur implique d'assumer son propre discours avec une position de sujet, un point de vue, un projet, une intention, quelle qu'elle soit, qu'il s'agisse de comprendre et expliquer le monde, d'agir sur autrui ou qu'elle soit d'ordre esthétique. La notion de discours, à cet égard, mérite d'être privilégiée. Elle implique en effet une interlocution: le discours, fût-il écrit, est adressé, il est réponse et attend une réponse. Il suppose aussi un interdiscours : il est redevable à d'autres auteurs, porte les traces d'autres discours. Bref, désigner les écrits comme des discours revient à insister sur leur caractère dialogique, au sens de V. Voloshinov (2010) et de M. Bakhtine (1984).

12 L'auteur n'écrit pas seul, qu'il soit d'ailleurs placé en situation collaborative comme dans l'étude que nous allons présenter ou en situation d'écriture individuelle. Il est tributaire des textes qu'il lit, auxquels il emprunte et dont on trouve les traces dans sa production (c'est ce qu'on appellera hétéroglossie après M. Bakhtine) et il se situe dans un réseau dialogique : sa production répond à une consigne (ici d'abord d'appartenance à un genre), se place dans la continuité du texte qu'il vient de lire (ici une suite de texte), anticipe des réactions de lecteurs sur lesquels il veut produire des effets, prend place dans une série de productions de pairs dont il a eu à faire une critique. L'auteur est un sujet «situé » au sein d'une communauté discursive qui est à la fois celle des partenaires avec lesquels il interagit et des lecteurs auxquels il s'adresse (dans notre cas la communauté scolaire et plus particulièrement la ou les classes) et celle des objets culturels socialement constitués (les discours, les documents, les livres...) avec lesquels il interagit d'une autre manière (Kostouli, 2009). 


\section{Un savoir écrire en devenir} d'écriture en distinguant d'une part le contexte des discours où un auteur (y compris un apprenti auteur) élabore son propre discours et d'autre part la compétence présente de l'auteur à procéder à cette élaboration, compétence en construction lorsqu'il s'agit de jeunes apprentis auteurs. Cette compétence est très inégalement partagée entre apprentis auteurs d'une même classe d'âge, et cette inégalité est marquée par l'origine sociale des élèves ${ }^{1}$. Ce sont non seulement les connaissances linguistiques qui sont inégales, mais aussi le rapport au langage écrit, la compréhension même de l'idée que l'écrit se travaille, est l'objet d'une élaboration cognitivo-langagière, et non pas l'outil « transparent » de la transcription de connaissances préexistantes (Bautier, 2005), et que, dans l'activité d'écriture, les discours des autres (et, abstraits de ces discours, les mots, les structures, les genres) sont des ressources dont il convient de s'emparer. Certains élèves acquièrent cette compétence facilement. Ce sont souvent ceux dont le rapport au langage construit de plain-pied avec la socialisation langagière familiale est "scriptural-scolaire", pour reprendre la terminologie de B. Lahire (2008), ou « secondarisé » si nous adoptons cette notion transposée par É. Bautier et J.-Y. Rochex (2004) de la notion bakhtinienne de « genres seconds ». D'autres élèves, qui entretiennent un rapport « oral-pratique » avec le langage écrit (Lahire, 2008) ont d'autant plus de mal à se forger une compétence d'auteur que celle-ci, si elle est sollicitée par les enseignants, est rarement accompagnée et explicitée par ceux-ci (Rochex \& Crinon, 2011). Travailler sur le déjà-là, ce n'est donc pas se résigner aux compétences déjà-là, lorsque ces compétences sont peu affirmées.

C'est pourquoi il convient de s'interroger sur la dynamique temporelle de l'appropriation du savoir écrire. Le "déjà-là » renvoie à la fois à des connaissances antécédentes à la nécessité de leur mobilisation. Ce sont les connaissances antérieures à mettre en lien avec de nouvelles connaissances. Ce sont aussi les connaissances "presque-là », non encore stabilisées, liées à des représentations approximatives ou erronées de ce que suppose la tâche. Il y manque en effet le temps de la construction sociale des savoirs et des pratiques langagières normées par les attentes et les contraintes de l'école, liées à des genres spécifiques, en rupture avec les caractéristiques de la plupart des pratiques langagières conduites hors de la classe.

Le cadre de référence vygotskien de la zone proximale de développement (Vygotski, 1997) permet de comprendre la manière dont le « déjà-là » entre en dialogue avec le « presquelà ». Les dimensions temporelles et spatiales s'y rejoignent, puisque l'espace de la circulation du savoir suppose le temps nécessaire à l'appropriation, au passage de la sphère de l'intersubjectif - lieu du dialogue avec autrui où la connaissance est extérieure et « pas encore là » - à l'intrasubjectif où cette connaissance est « à présent là », mobilisée dans la pratique d'écriture.

D'un élève à l'autre, la ligne de démarcation entre le «déjà-là » et «l'être-là » en construction est labile, peut faire l'objet d'adaptation ou de transformation, de déplacement... 


\section{Réagencer le « déjà-là » : étayage et mémoire externe}

17 La prise en compte de l'élève en tant que sujet de ses apprentissages dans son interaction avec l'environnement scolaire et extrascolaire est sous-tendue par l'idée que l'apprentissage nécessite l'étayage des enseignants, des ainés (Bruner, 1983, 1996; Vygotski, 1997) ou des pairs (Allal et al., 2005) pour les aider à atteindre l'objectif cognitif lié à la situation d'apprentissage et à résoudre les problèmes auxquels les confronte l'activité d'écriture. Il s'agit d'agencer et de réagencer des matériaux langagiers issus de productions antérieures effectuées par soi et par d'autres, issues de lectures proches mémorisées, pour les transformer.

Lors de la récriture de textes narratifs, les possibilités d'emprunt à des textes issus de sources différentes favorise la stratégie de "transtextualisation", que nous définissons comme l'appropriation par les élèves d'éléments phrastiques intégrés dans leurs textes cibles. La copie, qui est l'une des formes de l'emprunt, correspond, dans ce genre textuel scolaire, à ce que M.-C. Penloup (2007) nomme «la tentation du littéraire chez des scripteurs ordinaires ».

\section{Méthodologie de l'étude}

19 L'analyse qui suit cherche à illustrer la construction de la compétence d'auteur chez des apprentis scripteurs. Le corpus dont sont tirés les exemples analysés est constitué de productions d'élèves de $\mathrm{CM} 2$ ( $5^{\mathrm{e}}$ année de l'école primaire) de quatre écoles de la région parisienne.

Cent quarante-quatre élèves ont lu des romans d'aventures (Astafiev, 1986 ; Murail, 1998 ; Ollivier, 1995 ; Steig 2003), et ont écrit un épisode s'insérant dans certains de ces romans, à quatre reprises dans l'année, de novembre à juin.

21 Dans chacune des classes concernées, une séance inaugurale de présentation du dispositif a consisté à expliciter aux élèves les enjeux du projet et ses présupposés méthodologiques, afin d'éviter les malentendus liés à l'interprétation des consignes et à l'enrôlement dans l'activité. Cette séance a ainsi permis de définir, à partir d'exemples de textes d'auteurs de littérature de jeunesse, les caractéristiques d'un extrait de roman d'aventures réussi. Parallèlement a été conduite une phase d'initiation à la lecture critique de textes permettant à chaque élève de rédiger des conseils constructifs à l'adresse de ses pairs, dans le but de l'aider à améliorer sa première version d'écriture. Un entrainement à la réception de critiques et aux stratégies de prise en compte des conseils donnés a également été mis en œuvre afin de familiariser les élèves des différentes classes avec les activités de lecture et d'écriture atypiques auxquelles ils seraient régulièrement confrontés au cours de l'année scolaire.

Chaque séquence répondait ensuite au même déroulement, en quatre séances séparées par trois ou quatre jours. Les consignes d'écriture demandaient de placer le personnage face à différentes épreuves le conduisant à s'inscrire dans une succession d'actions. Ainsi, le héros, perdu dans la forêt, doit tour à tour affronter le froid et la faim dans le premier épisode, une bête sauvage, dans le deuxième, une tempête, dans le troisième et enfin, dans le dernier épisode, tenter d'échapper à des poursuivants. 
Chacune des activités rédactionnelles s'est trouvée insérée dans un continuum de lecture écriture. Chaque élève a ainsi rédigé, à quatre moments distincts de l'année scolaire, un épisode le conduisant successivement à (i) lire un extrait de roman d'aventures; (ii) écrire la suite de ce texte à partir d'une consigne; (iii) échanger par courriel avec plusieurs élèves d'une classe distante sur la manière d'améliorer ce texte ; (iv) récrire une nouvelle version du texte initial.

L'intérêt didactique du dispositif tient à la rupture qu'il introduit par rapport aux situations de classe habituelles où la correction des écrits d'élèves relève exclusivement de la compétence et par conséquent de l'autorité du professeur. Dans le dispositif décrit ci-dessus, est instaurée une collaboration entre pairs qui déplace les élèves de la "position basse» qu'ils occupent dans le rang des savoirs à une "position haute» (Sensevy, 2006), proche de celle qui définit a priori l'enseignant détenteur de connaissances. Lors de la séance consacrée à la communication électronique, les élèves de deux des quatre classes concernées ont lu plusieurs textes de leurs camarades et formulé par écrit des conseils et critiques à leur intention. Ils doivent ainsi endosser le rôle de l'expert. En revanche, les élèves des deux autres classes n'ont pas lu les textes de leurs pairs. Leur activité a consisté à prendre connaissance des remarques qui leur avaient été adressées et à y apporter une réponse. Quand ils reprenaient leur texte, ils étaient libres de les prendre en compte ou de les ignorer.

Le corpus d'étude est constitué des deux versions - écriture et récriture - des textes produits par les élèves lors de chacune des quatre séquences et des échanges (conseils et réactions à ces conseils) réalisés lors des troisièmes séances.

Une analyse statistique fondée sur la comparaison entre les versions 1 et 2 de chaque texte et entre les premières versions des quatre textes produits a mis en évidence une appropriation progressive, chez l'ensemble des participants, des caractéristiques du genre et du projet de produire sur les lecteurs les effets habituels auxquels ils prennent plaisir dans un roman d'aventures : exotisme du décor, identification au héros, attente et suspense, scènes d'action rythmées... Cette appropriation est significativement plus marquée chez les élèves qui ont lu et critiqué les textes de leurs pairs pour leur donner des conseils (c'est-à-dire les tuteurs) que chez ceux qui ont reçu des conseils (pour le détail de ces analyses, voir Crinon, 2012 ; Crinon \& Marin, 2010b). C'est pourquoi nous analyserons ici des exemples caractéristiques pris parmi les productions des tuteurs.

\section{La dynamique d'un apprentissage}

\section{Ce que sait faire Shirley en fin d'année}

27 Nous prendrons comme exemple le dernier texte de Shirley, qui illustre parfaitement ce que beaucoup d'élèves ayant bénéficié du dispositif avec le rôle de tuteur sont capables d'écrire en fin d'année ${ }^{2}$.

Pendant ce temps, Jeremy continuait à escalader posément les pentes du premier contrefort de la Cordillère. À peine fini d'escalader le premier contrefort, le fugitif pouvait tomber dans des sables mouvants. Cette forêt abritait énormément de sables mouvants, marigots, serpents et des veuves noires. Ce qu'il détestait prodigieusement.

L'évadé se rattrapa de justesse comme un équilibriste dans un arbre. Le garçon sauta par-dessus. Quand il arriva derrière le sable mouvant, il vit le garage où on rangeait les motos, les voitures qui servaient à transporter les cannes à sucre. 
Jeremy eut l'intelligence exceptionnelle d'emprunter une moto pour fuir plus rapidement ses poursuivants. Mais avant Jeremy eut des fourmillements dans les jambes. Il enleva son pantalon et trouva une veuve noire qui faisait le marathon à la recherche des blessures gorgées de sang. Le garçon l'attrapa et la jeta le plus loin. Après l'avoir jetée, il lécha sa piqûre. Ses poursuivants étaient de plus en plus proches du "gibier ». Dogue s'arrêta et hurla de douleur. Thompson regarda et constata que Jeremy avait semé des graines toxiques. «Le gredin, il a semé des graines toxiques » cria Thompson. «Comment va-t-on faire? » interrompit le chef surveillant.

Jeremy entra, mit le casque de moto et surgit du garage. Il arriva juste devant ses poursuivants. C'est comme ceci que continua la course poursuite. Jeremy pleurait de joie. Il avançait comme un guépard sur sa moto. Il dérapa. Ses poursuivants s'arrêtèrent. Jeremy s'assit dans le creux de l'arbre puis il commença à prendre congé. De leur côté les poursuivants progressaient de plus en plus. Ils parcouraient le chemin du fugitif. Arrivés en haut de la première Cordillère, les poursuivants ainsi que leur dogue enjambèrent les sables mouvants. D'un bond Jeremy se leva et dit : « Vous revoilà, vous m'avez suivi! »

Le fugitif lança des graines toxiques sur leurs têtes : "sale bâtard » Jeremy sauta de son arbre et la poursuite commença. Le fugitif en avait marre de courir. Il venait de se reposer une minute. A moitié hurlant, à moitié pleurant, il courait comme un guépard dans la boue. Les poursuivants suivirent les traces dans la boue. Jeremy grimpa dans un arbre. Les fugitifs couraient couraient toujours sans se soucier si l'évadé se serait caché dans un arbre. Le fugitif mangea des melons d'eau puis dormit au creux de l'arbre bien au chaud. (Shirley, séquence 4, version 2)

La comparaison avec sa première production, quelques mois plus tôt, permet de caractériser le chemin parcouru, au-delà des maladresses qu'on y observe.

Vassioutka alluma un feu puis il ramassa des branches d'arbres et des feuilles épaisses. Il commença à construire une cabane, ensuite, il fit un lit en feuilles qu'il glissa à l'intérieur de la tente. Soudain, il s'arrêta parce qu'il avait faim; il pensa à l'oiseau dans son sac. Vassioutka prit son oiseau avec quelques cônes de cèdre. Il dépluma l'oiseau, frotta les cônes, les fit cuire. Vassioutka finit de manger, il éteignit le feu puis il s'endormit et il pensa que le lendemain il retrouverait son chemin. (Shirley, séquence 1 , version 2)

Du point de vue de la quantité de texte produite, la progression des écrits de Shirley est représentative de l'ensemble des élèves tuteurs : le texte qu'elle rédige au mois de mai est quatre fois et demie plus long que celui d'octobre et la longueur du texte traduit le passage d'une simple succession d'actions à un enchevêtrement d'actions, d'états mentaux et d'éléments descriptifs.

30 En second lieu, la diversification des verbes utilisés au fil des versions et des séquences est un indice de la transformation du déjà-là en compétences rédactionnelles. Le texte produit en fin d'année utilise, non seulement davantage de verbes de mouvement permettant de donner un rythme à la poursuite (escalader, fuir, courir, tomber, attraper, jeter, se rattraper, sauter, arriver, avancer, déraper, s'arrêter, s'assoir, prendre congé, progresser, parcourir, enjamber, se lever, lancer, grimper, suivre), mais aussi des verbes descriptifs ( abriter, hurler de douleur, avoir des fourmillements), ainsi que d'autres verbes indiquant les états mentaux du personnage (détester, avoir l'intelligence de, pleurer de joie).

31 Sur ce second critère, l'exemple des différents textes écrits par Vuong est aussi très éclairant. L'évolution dans l'utilisation de verbes génériques par défaut, dont il faisait un large usage en début d'année, permet d'observer l'intensité des progrès effectués. En séquence 1 , les verbes employés - essentiellement le verbe faire - s'avèrent peu diversifiés et redondants : 
Vassioutka avait déjà mangé son pain. Mais il avait encore faim. Alors il se mit à courir dans la forêt lorsqu'il trouva des châtaignes. Il les fait griller. Il repartit dans la forêt, prit des bâtons.

Ensuite il retourna dans la clairière et fait déjà griller les châtaignes en fait du feu. La nuit tomba. Vassioutka avait fait son feu, il avait son fusil à côté de lui pour la nuit. (texte de Vuong écrit en septembre) douze verbes d'action. Certes, certains de ses emplois relèvent ici de la collocation ou sont auxiliaires; ils attestent néanmoins une forte attraction de l'élève pour ce verbe supragénérique qui évite de recourir à des verbes spécifiques, plus difficiles à solliciter en mémoire, voire absents de son répertoire lexical.

En revanche, dès la séquence 2, après avoir critiqué plusieurs textes, Vuong a considérablement enrichi son propre texte :

Vassioutka entendait des bruits. Il voyait une ombre dans la nuit. Cette chose avait des yeux comme des diamants dans la nuit. Cette ombre se rapprochait. Il avait très peur. Lorsque la chose se montra. Il aperçut un lynx. Il avait un pelage très magnifique avec des pattes grosses et velues. Vassioutka chargea son fusil. Il tira tellement vite qu'il ne voyait pas où il visait. Paralysé par le lynx, il courut dans la forêt. Lorsqu'il arriva au bord d'une chute d'eau, il décida d'affronter le lynx. Le lynx sauta sur lui. Vassioutka esquiva l'attaque de justesse. Mais hélas, il tomba avec le lynx dans la chute d'eau. Vassioutka avait quelques égratignures. Mais le lynx rejeté sur le bord ne se releva pas. Vassioutka prit son fusil et tira. Le lynx mourut. Et le triomphe revient à Vassioutka qui ramena la peau du lynx dans la clairière et s'en servit pour une couverture. (texte de Vuong, version 2 écrit en novembre) emploi des adverbes. Dans le texte de début d'année, aucun adverbe, stricto sensu, ne précise les phrases ou les éléments de phrase. En revanche, le texte de la quatrième séquence - et cela dès la première version ${ }^{3}$ - fourmille d'adverbes et de locutions adverbiales rendant des nuances variées, exprimant alternativement, et parfois simultanément, la manière (posément, de justesse, plus rapidement) la quantité (à peine, de plus en plus, énormément, prodigieusement), l'espace (juste, devant, le plus loin, de leur côté). La distribution habile de ces éléments du discours permet de spécifier l'action aussi bien que la description, à mesure que le jeune scripteur se forge un style. L'auteur fait participer le lecteur au spectacle de l'ascension de Jeremy « continua[n]t à escalader posément les pentes du premier contrefort de la Cordillère ». Le décor est planté, on suit pas à pas le protagoniste dans sa progression, et la manière dont il assure sa sécurité dans cet épisode de poursuite éprouvante.

Examinons enfin le point de vue narratif adopté. En fin d'année, Shirley choisit, pour tenir ses lecteurs en haleine, d'alterner le point de vue du poursuivi et celui des poursuivants. Les obstacles qui retardent la marche, tantôt du héros, tantôt de ses 
persécuteurs, sont ainsi communiqués aux lecteurs, permettant de faire se succéder soulagement et regain d'inquiétude pour le personnage auquel va leur sympathie.

\section{Ce qui était en germe dans sa production de début d'année}

La première écriture montre des compétences balbutiantes, qui témoignent cependant de savoir-faire liés à des représentations en cours d'élaboration de ce qu'est un texte de fiction et de ses caractéristiques.

Les énumérations fournissent un exemple particulièrement éclairant de la manière dont le « déjà-là » de la première séquence est mobilisé en fin d'année scolaire, pour s'installer en stratégies d'écriture maitrisées.

L'exemple ci-dessous présente une succession d'actions rendue par la juxtaposition de propositions elliptiques au sujet commun :

Il dépluma l'oiseau, frotta les cônes, les fit cuire. (Shirley, séquence 1)

Cette habileté se particularise pour créer un effet rhétorique dans la production d'écrit de la séquence 4 où le sémantisme du verbe courir se trouve renforcé par sa répétition, créant un rythme qui mime la course haletante :

Les fugitifs couraient couraient toujours sans se soucier si l'évadé se serait caché dans un arbre. (Shirley, séquence 4)

Puis l'élève applique l'énumération qui devient accumulation au domaine des substantifs et groupes nominaux qui font ainsi se dessiner un paysage animé au fil des mots :

Cette forêt abritait énormément de sables mouvants, marigots, serpents et des veuves noires (Shirley, séquence 4)

Dans un autre registre sémantique, le même procédé d'énumération s'étend ensuite aux noms des véhicules, montrant par l'usage du pluriel grammatical et du détail des catégories mentionnées l'abondance du parc automobile :

il vit le garage où on rangeait les motos, les voitures (Shirley, séquence 4)

$4 \mathrm{Au}$ fil des textes que réalisent les élèves tuteurs au cours du protocole mis en place, savoirs et savoir-faire se complexifient. Il semble que leurs représentations initiales approximatives - des propriétés de l'écriture littéraire s'affinent ensuite et leur permettent d'imiter à bon escient ce qu'ils trouvent dans leurs lectures.

\section{Ce qui a fait ressource dans les textes qu'elle a lus}

Différentes ressources, lexicales, mais aussi syntaxiques et rhétoriques, sont accessibles à partir des textes lus par les élèves.

La mention des «fourmillements» qui relève d'un registre littéraire dans le texte de Shirley est empruntée au récit de Jean Ollivier, dans Le cri du kookabura (Ollivier, 1995). La métaphore «des dizaines de sangsues [qui] se gorgeaient de son sang » dans ce même texte d'auteur donne lieu à une dépronominalisation du verbe pour aboutir à la création originale de la jeune élève : « une veuve noire qui faisait le marathon à la recherche des blessures gorgées de sang ».

Les comparaisons sont aussi récurrentes dans les textes ressources mis à la disposition des élèves, et dont Shirley a pu tirer profit. L'auteur du Cri du kookabura dit du héros qu'il «frissonnait comme un tremble », que le chien «bavait comme un fou furieux ». L'auteur évoque «un gourdin poli comme de l'os». Alain Surget (2002), dans l'extrait de son 
roman Le fils des loups, emploie l'expression « détaler comme un lapin ». Dans Pas de whisky pour Méphisto, Paul Thiès (1991) caractérise ainsi Microbe : « discret comme une souris ».

Ces comparaisons réitérées dans les textes qu'elle a lus ont fait ressource pour Shirley. Les expressions suivantes mettent en scène de telles associations entre comparant et comparé :

L'évadé se rattrapa de justesse comme un équilibriste dans un arbre

Le sème commun au comparé et au comparant dans l'image employée par la jeune auteure est celle de l'instabilité, assez proche sémantiquement du frisson de l'arbre, dont on peut faire l'hypothèse qu'il a pu inspirer l'orientation de la comparaison.

La deuxième comparaison inventée par Shirley prend également sa source dans un texte d'auteur. Lorsqu'elle écrit :

Il avançait comme un guépard sur sa moto

51 sa création lexicale consiste à substituer un animal, le guépard, à un autre, le lapin. Le sème commun est la rapidité, associée à un verbe de mouvement : «détaler » devient "avancer », cependant la moto implique une accélération du déplacement et justifie pleinement la substitution du guépard au lapin. La reprise de l'image montre l'insistance sur la progression au cours de la poursuite et la volonté de filer la comparaison :

il courait comme un guépard dans la boue

52 Or rien de tel n'apparaissait lors de la séquence 1, au début de l'année scolaire (voir supra, texte de la séquence 1 ).

53 L'usage de l'interrogation comme moyen de faire surgir le suspens est fortement présent dans Le fils des loups : «La piste de la Bête rejoignait la sienne, lui coupait la retraite. Mais où était-elle? Devant lui ? Derrière ? Tapie sur un côté ?».

Les interrogations enchainées miment l'inquiétude du protagoniste, qui cherche d'où viendra la menace. De la même manière, Shirley utilise ce procédé dans son dernier texte :

«Le gredin, il a semé des graines toxiques », cria Thompson. «Comment va-t-on

faire?» interrompit le chef surveillant.

Elle s'approprie le procédé en donnant à entendre le désarroi et la colère du chef des poursuivants, en la faisant exprimer à voix haute. Cette technique narrative, acquise au fil des séquences du dispositif et des lectures successives était peut-être en germe au début de l'année où elle évoquait les pensées du héros :

il pensa à l'oiseau dans son sac

et il pensa que le lendemain il retrouverait son chemin.

56 On voit l'évolution entre l'évocation des pensées des personnages au discours indirect et le passage au discours indirect libre auquel parvient Shirley en fin d'année.

\section{Devenir tuteur}

\section{Du mimétisme professoral à l'autonomie de jugement}

Les critiques élaborées par les élèves tuteurs fournissent une trace de l'évolution des compétences intuitives - presque-là - en compétences qui s'installent. Prenons l'exemple de l'évolution des critiques et conseils formulés par Stéphane entre la deuxième et la quatrième séquence. 

matrice qui se développe ; les remarques sont similaires et semblent mécaniques. Ainsi des commentaires de Taylor :

Tu n'as pas décrit l'aigle. Tu as bien décrit le combat. Tu n'as pas décrit le sentiment de Vassioutka. (Taylor, séquence 2 à Mamou)

Tu décris bien le combat. (Taylor, séquence 2 à Steven)

Tu ne décris pas comment est l'animal. (de grosses pattes...) (Taylor, séquence 2 à

Anthony)

Décris mieux ton aigle. (Taylor, séquence 2 à Étienne)

Les remarques proposées au mois de décembre indiquent déjà une compréhension des enjeux et une attention particulière à la tâche demandée. L'élève tuteur prend en charge les difficultés et les manques qu'il constate dans les textes de ses pairs pour les aider à identifier ce qui peut être amélioré.

Ton texte est bien mais une torche ne devrait pas faire fuir un ours géant.

Il n'a pas le temps de fabriquer une torche pendant le combat (Stéphane, séquence

2, conseil à Loïc)

long de l'année.

Un crocodile ne se chasse pas avec un bâton

Le crocodile n'a pas peur des bâtons (Stéphane, séquence 4, conseil à Loïc) comme la marque de l'appropriation du contrat de lecture propre au roman d'aventures, régi par les codes du vraisemblable. On voit comment les connaissances « déjà-là » issues de l'expérience personnelle et de l'univers des lectures permettent de construire un point de vue de critique.

de l'enseignant. Les conseils prescriptifs donnés en début d'année semblent également mimer le discours professoral en soulignant les manques :

Tu n'as pas assez donné de détails sur le combat. Il n'est pas entier (Stéphane,

séquence 2, conseil à Junior)

susceptibles de faire comprendre aux élèves dont les textes sont critiqués l'intérêt des améliorations implicitement suggérées :

A quoi servent les deux melons ? (Stéphane, séquence 4, conseil à Junior)

Court-il juste pour des moustiques? (Stéphane, séquence 4, conseil à Tiffaine)

\section{De la répétition à la diversification}

4 La focalisation sur la description intervient de manière répétitive, sans fournir de précisions pouvant aider les élèves critiqués. Cependant, au fil des séquences, les compétences de Taylor en matière de critiques s'affirment et celles qui étaient en devenir adviennent. En séquence 4, les remarques de Taylor se diversifient :

Tu n'expliques pas trop bien. Dans un roman d'aventure le héros n'est pas mort.

Dans le texte il y a la rivière. (Taylor, séquence 4, à Mamou)

Il faut de la poursuite. (Taylor, séquence 4, à Etienne)

65 La variété des conseils montre une émancipation de Taylor qui cesse de «singer » ce qu'il a retenu du discours de l'enseignant, pour entrer dans la spécificité des textes qui lui sont 
soumis. Ce changement de posture l'entraine ainsi vers le "méta ». Il le conduit à se projeter dans le texte d'autrui pour proposer des formulations alternatives illustrant par l'exemple les consignes générales qu'il donne :

Tu peux mettre : "Son poignard fendit l'air et Jeremy poussa un cri. La pointe lui heurta l'épaule, taillada sa chemise et lui traça une longue estafilade sur sa peau. » (Taylor, séquence 4, à Mamou)

C'est bien ton texte mais tu n'as pas parlé des sentiments de Jeremy. Tu pourrais mettre «les poursuivants voient les traces de Jeremy». (Taylor, séquence 4, à Steven) faire progresser dans leurs compétences à écrire: les travaux en didactique de la production de textes des années 80 et 90 ont mis l'accent sur ce point. On voit ici en quoi c'est un puissant levier de progrès, dès lors qu'il ne s'agit pas seulement de corriger des écarts à la norme linguistique. Les jeunes scripteurs dont nous avons ici analysé les productions et le travail combinent deux types de déjà-là, ce qu'ils savent déjà faire ou presque faire (dans le cas analysé, énumérer des actions, produire une suite chronologique) et ce qu'ils empruntent aux autres, les experts et les pairs (dans le cas analysé, le procédé d'accumulation, les adverbes, le procédé de jeu sur les points de vue du poursuivi et des poursuivants, les images), pour gagner en efficacité, et écrire un texte plus proche des caractéristiques du genre pratiqué, plus à même de produire sur les lecteurs le plaisir de lecture qu'on attend d'un roman d'aventures. Un «catalyseur » de cette alchimie d'un déjà-là interne et d'un déjà-là externe, est, dans la situation observée, le travail métacognitif qui accompagne la transformation de ces élèves en tuteurs de leurs pairs. Pour dire les choses autrement, si les apprentissages constatés ici peuvent avoir lieu, c'est parce que l'appropriation des ressources tirées des lectures peuvent s'ancrer sur des connaissances et des savoir-faire antérieurs, parce qu'elles sont "presque-là ». Mais ce passage du presque-là à la compétence manifeste ne va pas de soi. Elle nécessite, pour beaucoup d'élèves, et en particulier les plus scolairement fragiles, que des 
conditions didactiques particulières soient réunies: permettre de faire interagir les élèves avec les textes et entre eux fait partie de ces conditions favorables.

En outre, ces transformations vont de pair avec une clarification des connaissances et des ressources présentes mais « ignorées » (Penloup, 2007). Construire des connaissances qui vont devenir des ressources mobilisables dans l'activité passe aussi par la construction de connaissances métacognitives, qui indiquent ce qui se joue dans les apprentissages, audelà du type de savoirs qu'ils engagent. Fait ressource ce qui sert à apprendre, à comprendre ce que l'on apprend et à apprendre ce que l'on comprend, pour appréhender, c'est-à-dire saisir ensemble et dans leur ensemble les composantes hétérogènes de l'écriture. Ainsi, dans le travail de tutorat, les élèves comprennent, non seulement les critères du genre pratiqué, mais encore plus fondamentalement qu'ils peuvent se servir des mots des autres et comment ils peuvent en faire usage, à partir de leurs connaissances "déjà-là », de la manière dont ils les mettent en relation avec celles que l'école et l'expérience quotidienne leur apportent.

\section{BIBLIOGRAPHIE}

ALlAL, L., MOtTIER LOPEZ, L., LeHrauS, K. \& FORGET, A. (2005). « Whole-Class and Peer Interaction in an Activity of Writing and Revision ». In : Kostouli, T. (éd.), Writing in context(s): Textual practices and learning processes in sociocultural settings. New York : Springer, p. 69-91.

ASTAFIEV, V. (1986). Perdu dans la taïga. Paris : Flammarion.

BAKHTINE, M. (1984) [1952]. Esthétique de la création verbale. Paris : Gallimard.

BAUTIER, É. (2001). «Pratiques langagières et scolarisation ». Revue française de pédagogie 137,

p. $117-162$.

- (2005). « Mobilisation de soi, exigences langagières scolaires et processus de différenciation ».

Langage et société 111, p. 51-71.

BAUTIER, É. \& RocheX, J.-Y. (2004). « Activité conjointe ne signifie pas significations partagées ». In : Moro, C. \& Rickenmann, R. (dirs), Situation éducative et significations. Bruxelles : De Boeck, p. $197-220$.

BIEMILLER, A. \& SLONIM, N. (2001). « Estimating root word vocabulary growth in normative and advantaged populations: Evidence for a common sequence of vocabulary acquisition ». Journal of Educational Psychology 93(3), p. 498-520.

BRUNER, J. S. (1983). Le développement de l'enfant : savoir-faire, savoir-dire. Paris : Presses universitaires de France.

- (1996). L'éducation, entrée dans la culture. Paris : Retz.

CRINON, J. (2012). « The dynamics of writing and peer review at primary school ». Journal of Writing Research 4(2), p. 121-154. En ligne : http://www.jowr.org/articles/vol4_2/

JoWR_2012_vol4_nr2_Crinon.pdf. 
CRINon, J. \& Legros, D. (2002). « The Semantic Effects of Consulting a Textual Data-Base on Rewriting ». Learning and Instruction 12(6), p. 605-626.

CRINON, J. \& MARIN, B. (2010a). « The role of peer-feedback in learning to write explanatory texts: Why the tutors learn the most ». Language Awareness 19(2), p. 111-128.

- (2010b). « Réviser à distance pour apprendre à écrire des textes narratifs ». Revue française de Linguistique appliquée XV(2), p. 85-99.

- (2012). «Rendre visibles les objets de savoir dans l'acquisition de compétences discursives». In : Beckers, J., Crinon, J. \& Simons, G. (éds), Approche par compétences et réduction des inégalités entre élèves : de l'analyse des situations scolaires à la formation des enseignants. Bruxelles : De Boeck, p. 17-32.

- (2014). « Point de vue et activité des élèves en production écrite ». In : Daunay, B. \& Dufays, J.-

L. (éds), Du côté des élèves. Bruxelles : De Boeck, p. 35-49.

FLORIN, A. (1999). Le développement du langage. Paris : Dunod.

HART, B. \& RISLEY, R. T. (1995). Meaningful differences in the everyday experience of young American children. Baltimore: Paul H. Brookes.

HAYES, J. R. (1996). « A new framework for understanding cognition and affect in writing ». In :

Levy, C. M. \& Ransdell, S. (éds), The science of writing. Mahwah, NJ : Lawrence Erlbaum Associates, p. 1-28.

- (2011). « Kinds of Knowledge-Telling: Modeling early writing development ». Journal of Writing Research 3(2), p. 74-92.

Kostouli, T. (2009). « A sociocultural framework: Writing as social practice ». In: Beard, R., Myhill, D., Riley, J. \& Nystrand, M. (éds.), The Sage handbook of writing development. London : Sage, p. 98-116.

LAHIRE, B. (2008). « L'inégalité devant la culture écrite scolaire : le cas de l'“expression écrite” à l'école primaire ». In : Lahire, B., La raison scolaire. Rennes : Presses universitaires de Rennes, p. 109-125.

LEE, Yon Ok, KRASHEN, S. \& GRIBBons, B. C. (1996). « The effect of reading on the acquisition of English relative clauses ». ITL International Journal of Applied Linguistics 113-114, p. 263-273.

LEGROS, D., CRINON, J. \& MARIN, B. (2006). « Réécrire et apprendre à réécrire : le rôle d'une base de données textuelles ». Langages, ${ }^{2} 164$, p. 98-112.

LIEURY, A. (1996). « Mémoire encyclopédique et devenir scolaire : étude longitudinale d'une cohorte sur les quatre années du collège français ». Psychologie et psychométrie 17(3), p. 33-44.

MARIN, B. \& CRINON, J. (2014). « Stéréotypes et contraintes de genres : quelles ressources pour la production de textes explicatifs et de fiction à l'école élémentaire? » Éducation et didactique 8(2), p. 39-58.

MURAIL, M.-A. (1998). Le chien des mers. Paris : L'École des loisirs.

OLLIVIER, J. (1995). Le cri du kookabura. Paris : Casterman.

PENLOUP, M.-C. (dir.) (2007). Les connaissances ignorées : approche pluridisciplinaire de ce que savent les élèves. Lyon : INRP.

Richard-Principalli, P., Ferone, G. \& CRINON, J. (2012). « Acquisition de compétences rédactionnelles et de connaissances sur les genres dans un dispositif d'interactions écrites ». In : Rivière, V. (éd.), Spécificités et diversité des interactions didactiques. Paris : Riveneuve Éd., p. 151-166. 
RochEX, J.-Y. \& CRINON, J. (dirs) (2011). La construction des inégalités scolaires. Au cour des pratiques et des dispositifs d'enseignement. Rennes : Presses universitaires de Rennes.

SCARDAmAlia, M. \& Bereiter, C. (1987). « Knowledge telling and knowledge transforming in written composition ». In: Rosenberg, S. (éd.), Advances in applied psycholinguistics, Vol. 2. Reading, Writing and Language Learning. Cambridge, MA : Cambridge University Press, p. 142-175.

SÉNÉCHAL, M. (2000). « Examen du lien entre la lecture de livres et le développement du vocabulaire chez l'enfant préscolaire ». Enfance 53(2), p. 169-186.

SENSEVy, G. (2006). «L'action didactique, éléments de théorisation ». Théma, Revue suisse des sciences de l'éducation 28(2), p. 205-226.

STEIG, W. (2003). L'Ile d'Abel. Paris : L'École des loisirs.

SURGET, A. (2002). Le fils des loups. Paris : Rageot éditeur.

THIÈs, P. (1991). Pas de whisky pour Méphisto. Paris : Syros.

Voloshinov, V. N. (2010) [1929]. Marxisme et philosophie du langage. Limoges : Lambert-Lucas.

VYGotski, L. (1997) [1934]. Pensée et langage. Paris : La Dispute.

\section{NOTES}

1. Sur les inégalités de connaissances lexicales et leur lien avec le milieu sociofamilial, voir A. Florin (1999); B. Hart \& R. T. Risley (1995); A. Lieury (1996). Sur les inégalités des performances dans les activités rédactionnelles, voir B. Lahire (2008). Le lien entre socialisation familiale et pratiques langagière est étudié par É. Bautier, 2001 à partir d'une synthèse de la littérature.

2. L'orthographe a été normalisée.

3. En séquence 4 la plus grande partie du texte définitif de Shirley est rédigée dès la première version d'écriture. C'est aussi une marque de ses progrès.

\section{RÉSUMÉS}

Cette étude vise à montrer l'effet de différentes catégories de ressources textuelles sur les compétences d'écriture narrative d'élèves de la fin de l'école élémentaire. En appui sur des modèles psychologiques de l'écriture et sur la notion de dialogisme, nous mettons à l'épreuve l'hypothèse selon laquelle l'emprunt à des textes lus permet aux élèves d'appréhender certaines contraintes de l'écriture de fiction. Les participants ont écrit et réécrit un épisode de roman d'aventures, à quatre reprises au cours d'une année scolaire. Ils devaient en outre donner des conseils à leurs pairs entre l'écriture de leur première version et la seconde. L'analyse de textes d'élèves représentatifs de l'ensemble des productions réalisées montre la récurrence de réemplois de mots et d'expressions issus d'un univers référentiel commun. Elle met également en évidence les stratégies (tremplin, levier, image, jeu sur le signifiant/signifié) utilisées pour s'emparer des mots des autres et les reconfigurations opérées dans le contexte nouveau de leur 
écrit personnel. La possibilité de puiser à différentes sources textuelles - textes d'experts ou d'écrivains novices - joue un rôle d'étayage dans l'apprentissage de la littératie. En outre avoir à produire un métadiscours critique sur les textes de pairs conduit à abstraire les principes de l'imitation créative.

This study aims at showing the effect of various categories of textual resources on the skills of students' narrative writings at the end of primary school. According to psychological models of writing and to the notion of dialogism, we hypothesize that borrowing from texts which are read by students leads them to master certain constraints of the writing of fiction. The participants wrote and rewrote an episode of an adventure novel, on four occasions during a school year. Moreover, they had to give advice to their peers between the writing of their first draft and the second one. The analysis of students' texts, which are representative of all the productions that have been realized, shows the recurrence of re-use of words and expressions stemming from a common reference universe. It also highlights the strategies (springboard, lever, image, playing with signifier/signified) used to seize words of others and reconfigurations operated in the new context of their personal paper. The possibility of drawing from various textual sources-texts of experts or writers novices-plays a role of propping up in the apprenticeship of the literacy. Besides, having to produce a critical "métadiscours" on peers' texts leads to abstract the principles of creative imitation.

INDEX

Mots-clés : écriture, emprunts, lexique, récriture, travail collaboratif

Keywords : writing, borrowing, lexicon, rewriting, collaborative work

\section{AUTEURS}

\section{BRIGITTE MARIN}

Université Paris Est, Circeft, EA 4384, Université Paris 8, UPEC, F-94000, France

\section{JACQUES CRINON}

Université Paris Est, Circeft, EA 4384, Université Paris 8, UPEC, F-94000, France 